\title{
Histoire des migrations et ethnicité à partir d'une réflexion en Asie du Sud-Est
}

Vers une anthropologie des frontières?

The history of migration and ethnicity from a South East Asian perspective.

Towards an anthropology of borders?

Jacques Ivanoff

\section{OpenEdition}

Journals

Édition électronique

URL : http://journals.openedition.org/transcontinentales/791

DOI : 10.4000/transcontinentales.791

ISBN : 978-2-7351-1557-0

ISSN : 1775-397X

Éditeur

Editions de la maison des sciences de l'homme

Édition imprimée

Date de publication : 31 décembre 2010

ISSN : 1950-1684

Référence électronique

Jacques Ivanoff, « Histoire des migrations et ethnicité à partir d'une réflexion en Asie du Sud-Est »,

Transcontinentales [En ligne], 8/9 | 2010, document 6, mis en ligne le 31 décembre 2010, consulté le 08 septembre 2020. URL : http://journals.openedition.org/transcontinentales/791 ; DOI : https://doi.org/ 10.4000/transcontinentales.791

Ce document a été généré automatiquement le 8 septembre 2020

Tous droits réservés 


\section{Histoire des migrations et ethnicité à partir d'une réflexion en Asie du Sud-Est}

Vers une anthropologie des frontières?

The history of migration and ethnicity from a South East Asian perspective. Towards an anthropology of borders?

Jacques Ivanoff

«Il faut repenser la notion de frontière pour essayer de comprendre les contradictions qui affectent l'histoire contemporaine. » (Augé 2009 :

1 Aujourd'hui, la globalisation, pensée par les grands organismes internationaux (Banque asiatique de développement (BAD), Banque mondiale, Nations unies, Unesco...) définit certaines règles que tentent d'appliquer les organismes régionaux comme l'Association des nations de l'Asie du Sud-Est (ASEAN) dans des domaines aussi variés que la gestion du patrimoine, les flux de personnes, les contrôles des frontières... Une contradiction évidente apparait immédiatement: celle d'un monde idéalisé, sans frontières, où les biens (plus que les personnes) pourraient circuler librement, une vision qui s'oppose à la réalité des politiques étatiques et des volontés «nationalistes" qui considèrent le contrôle des territoires comme une nécessité vitale, sans pour autant pouvoir se passer des flux migratoires. Il existe donc un discours, celui de la volonté politique de fluidifier les frontières et de régulariser les abus, et une pratique, celle du trafic humain aux frontières. Des millions de personnes les passent avec leur argent, leur sang, leur virginité et leur vie. L'ethnologie se doit alors d'apporter quelques lumières sur ces trafics en observant l'ancrage historique des réseaux et leurs structures, fondées sur l'histoire, les relations familiales et les rapports de forces au sein d'un même groupe. Quand les grands organismes nationaux et internationaux et les défenseurs de l'idéologie libérale créent des "marchés de frontières " (notamment en Thaïlande, à partir de 1991 et jusque vers 1998) chargés de pénétrer les pays autrefois communistes 
(Cambodge, Vietnam, Laos) ou les pays «à risque » comme la Birmanie, il s'agit de construire une base concrète pour assurer la domination économique de l'ancien bloc de l'Ouest qui échange des marchandises pour la reconstruction et le développement contre des ressources naturelles et de la main-d'œuvre. Pour la Thaïlande, l'exemple du marché frontalier Rong Klua (avec Poipet au Cambodge) est très représentatif. Ce n'est qu'une "usine" que font tourner 100000 Khmers. Ce marché qui se développe en cercles de plus en plus étendus et entraîne des stratégies socioéconomiques de survie remarquables, s'inscrit dans une histoire plus ancienne qui liaient les Khmers rouges aux Thaïlandais et à «la ligne de camps de réfugiés » qui formaient autrefois la frontière $e^{\mathrm{i}}$, une structure que l'on retrouve aujourd'hui chez les réfugiés frontaliers karen. L'ethnologie des frontières ne peut donc se départir de l'histoire car cette dernière a créé les grandes fissures et les coupures entre peuples, celles provoquées par la colonisationbien sûr, mais aussi celles nées du flou des frontières. Ces lignes tampon et d'échanges sont des lieux d'expression de la fluidité ethnique, et donc des choix sociaux et culturels (Benjamin 1988; Scott 1990, 2009). Elles participent à la construction idéologique nationale mais aussi à l'ethnicité régionale, par exemple entre le Kelantan malais et l'ancien sultanat de Patani divisé en plusieurs provinces thaïlandaises (Horstmann 2002; Ivanoff 2010). Les deux segments sont issus d'une matrice malaise commune avant que l'endocolonialisme siamois et l'émergence de la Thaillande contemporaine ne viennent couper la région ; mais, ils ne suppriment pas les liens préexistants, bien au contraire, ils les fluidifient et créent de nouvelles formes d'organisations sociales. L'endocolinalisme est ici un moyen de gérer les frontières si tant est qu'on leur laisse une liberté d'expression fondée sur des réseaux régionaux.

2 Les marchés des frontières sont apparus en Thaïlande avec les premiers gouvernements "démocratiques", c'est-à-dire élus (même si les élections sont dominées par un clientélisme structurel à la nation). Ils n'ont pas permis de développer les pays qui devaient rattraper leur retard socioéconomique mais ont au contraire renforcé l'emprise des pays « riches » de la région sur les pays "pauvres » (Murshid et. al.2005). Le déséquilibre institutionalisé et économiquement indestructible, l'appel d'air provoqué par le développement et donc le manque de main-d'œuvre (Boutry et Ivanoff 2009) ont renforcé l'illégalité en dépit de certains efforts de régularisation et de législation. Et, plus les grands organismes tentent de construire des ponts, des ports, des routes, des corridors (Leveau 2009 ; Tréglodé et Leveau 2010), plus ils favorisent les migrations illégales. Rappelons également que la Thaïlande compte environ 5 millions (si ce n'est le double) de travailleurs illégaux qu'elle arrive à intégrer car elle en a besoin et que c'est aussi sa seule chance de se maintenir en tant que nation. Mais, à terme, cela pose des problèmes, comme celui de la colonisation adaptative des Birmans qui ont fait du sud de la Thaïlande une marche pour aller plus en avant.

\section{L'anthropologie des frontières : un discours moderniste sur la relation nomades/sédentaires}

3 Nous développons une épistémologie de l'anthropologie des frontières à l'intérieur de projets de recherche en coursii. Nous mentionnons Benedict Anderson et ses communautés imaginées, Georges Condominas et ses espaces sociaux qui permettent de concevoir des espaces de réflexion mouvants, Fernand Braudel et le désenclavement planétaire... Nous nous intéresserons ici succinctement à Fredrik Barth (dont nous 
avons déjà amplement parlé (Boutry et Ivanoff 2008) et James Scott qui ont tous deux permis de comprendre que les ethnies ou groupes sociaux ne survivaient et ne s'adaptaient que dans l'interaction, c'est-à-dire dans une adaptation perpétuelle à des objectifs culturels déclarés. Mais ces innovations sont aujourd'hui quelque peu dépassées. Depuis que les choix nomades (Benjamin 1988; Ivanoff 2001) définis et analysés selon des concepts anthropologiques sont apparus comme une évidence, le Zomia (territoire découpé arbitrairement et dans lequel les populations rejetant les États vivraient selon des codes culturels propres à favoriser leur résistance) de Scott apparaît comme une bonne hypothèse. En effet, il renverse la hiérarchie des notions pour une analyse plus nuancée et contrastée des rapports entre États et minorités. Rappelons pourtant que ces zones dites « hors système » étatique sont présentes depuis que les États existent car il n'y a pas de nation composée d'illusoires populations "sédentaires" sans populations nomades ou périphériques, les unes étant une nécessité pour les autres (elles créent de l'idéologie, des essais sociaux et apportent en temps de crises des solutions...) ; leur relation étant organique, la théorie de Scott n'a pas de fondements historiques et archéologiques suffisants pour construire une théorie fondamentale, ses écrits restent pourtant un stimulant intellectuel nécessaire. Le nomadisme périphérique bouge géographiquement et culturellement avec les États " sédentaires ». Dès lors, Scott n'a rien découvert d'autre que ce que les anthropologues avaient déjà inscrit sur leurs tablettes méthodologiques. Quant à Barth, il a certes compris qu'une ethnie ne pouvait s'appréhender que dans un ensemble de relations et que les interrelations créent la dynamique sociale. Il a seulement posé une hypothèse sans répondre à la question de savoir quel était le niveau limite d'interaction nécessaire pour faire disparaître une ethnie ou un groupe. Il ne pose pas non plus la question de savoir quelles sont les conséquences lorsqu'un groupe social prend le pas sur une ethnie, ou inversement, quand une ethnie réorganise un segment social d'une société dite sédentaire. Nous avons montré (Ivanoff 2004 ; Boutry et Ivanoff 2008) que les ethnies ont alors un rôle essentiel à jouer dans l'organisation du territoire (elle connaissent les ressources, elles savent les exploiter, elles connaissent les esprits et les puissances de l'au-delà) que les peuples « dominants » ne peuvent éluder (Ferrari 2008). Les pêcheurs birmans ont besoin des Moken pour s'adapter à la mer qu'ils ne comprennent pas d'où la naissance d'un syncrétisme culturel dans le Ténasserim; de même, dans le sud-ouest de la Thaïlande, une cosmologie interethnique s'est créée autour de rituels rappelant le partage des ressources et de la place de chaque population, donnant aux nomades marins une importance démesurée par rapport à leur rôle dans l'économie de la région. Aux frontières, la tradition d'intégration et de création de compromis, de syncrétismes et de rééquilibrages, continue de perdurer sans que l'État, aussi présent soit-il, ne puisse rien y faire.

4 Aujourd'hui, ces relations entre nomades et sédentaires s'inscrivent dans le cadre plus descriptif et moderne des concepts de centres et de périphéries. L'ethnicité ne disparait pas complètement, mais les groupes ethnonationaux et les groupes ethniques se mêlent. Un autre type de mobilité entre le centre et la périphérie apparaît avec l'émergence des États qui tentent de rationaliser leurs relations avec l'Autre. Ainsi, Alexander Horstmann (2002) pense que les nouvelles relations politiques et religieuses déterminent celles entre les peuples des frontières. Ceci n'est le cas que pour ceux qui sont séparés de leurs matrices et revendiquent une identité culturelle qui pourrait parfois rappeler la formation d'ethnies. Birmans colonisateurs dans le sud de la Thaïlande, Malais de Patani coupés de leur matrice créent ainsi des segments sociaux 
qui peuvent potentiellement s'ethniciser. Inversement, les ethnies (Moken et autres populations de nomades marins) semblent pouvoir s'intégrer dans ces segments sociaux : Moken de Birmanie qui se marient systématiquement avec des Birmans, mais aussi Moken de Thaïlande "traditionalistes" créant, ainsi, deux sous-groupes à l'intérieur d'une même ethnie. Dans la segmentation sociale et ethnique et la mixité entre le social et l'ethnique révélées par les frontières - et dans l'esprit politique des dirigeants des nations par la colonisation des fronts pionniers -, se manifestent les résiliences et les capacités d'adaptation, à l'origine des organisations sociales qui régissent aujourd'hui encore les moyens de production et les relations interethniques mais, également, les relations des régions aux États centralisateurs. À la suite de la confrontation pour l'exploitation de ressources apparaît la puissance de l'ethnicité.

\section{Le glissement politique de la réflexion anthropologique}

Il ne faut surtout pas réduire l'historique des relations nomades et sédentaires, et celui des relations séculaires entre peuples de frontières et «maîtres » relatifs des centres à des seuls conflits d'intérêts. Ainsi, Horstmann, un pionnier dans l'étude des populations des frontières, reste très politique et s'attache trop à la description des mouvements contemporains dans le traitement des questions actuelles posées par l'adaptation aux frontières, limitant ce "problème » à de simples questions de conflits de territoires, de ressources ou de religions.

The shocking escalation of violence in many borderlands has brought hidden geographies of the state into the limelight of the world's media and academia. Nevertheless, many of these regions remain virtually unknown. In the borderland of Bangladesh, Burma, and India-one of the least known places in South and Southeast Asia-the protest by Bangladeshi, Burmese, and Indian people against gas exploitation, forced labor, and loss of resources and land has come to the attention of a global public. But the atrocities committed by the Tatmadaw (Burmese army) in the Karen, Shan, and Rohingya borderlands and the consequent flight of the masses into neighboring borderlands remains virtually unnoticed. (Horstmann $2006: 3$ )

Il n'y a pas d'escalade mais une reconnaissance internationale et donc une redécouverte. Quelles que soient les conditions dramatiques décrites par les organismes internationaux, la situation était certainement pire avant que l'on reconnaisse ces drames ethniques. Mais, ces peuples résistent et il faudrait alors expliquer que les racines profondes de leurs "comportements ethniques", la base de leur ethnicité, puisent dans les guerres ou, en Birmanie, l'isolationnisme, le refus de s'ouvrir (qui permet une mainmise sur sa population plus importante qu'ailleurs) et des moyens puissants de résistance. Ici, l'interaction créatrice d'ethnicité de Barth peut redevenir un outil conceptuel pour peu qu'on lui reconnaisse une limite, celle de la survivance nécessaire d'un noyau ethnique liant des groupes entiers qui se sont construits des caractéristiques culturelles communes au cours de leur longue histoire.

7 Évidemment, les frontières "souples» de la Birmanie, les ethnies formant un "cordon" élastique protégeant le centre, ne sont pas les frontières "poreuses" du Cambodge et $\mathrm{du}$ Laos. La longue frontière birmano-thaïlandaise facilite le renfermement, les Birmans étant cernés par toute une ceinture d'ethnies plus ou moins indépendantistes et manipulées. La reconnaissance internationale et la "mobilisation » des intellectuels ne changent rien mais, par contre, recomposent le réel en 
communautés recréant des critères ethniques que les populations chassées et réfugiées sur les frontières connaissaient et adaptaient à leurs objectifs depuis très longtemps. Ces modes de gestion fondés sur des forces ethniques et des résiliences culturelles réagissant au développement des États n'ont pas attendu les chercheurs pour exister.

8 L'anthropologie des frontières, après avoir commencé en tant que domaine particulier, redevient un instrument d'analyse et d'évaluation dans les relations internationales. Plus on étudie le phénomène, plus on le fige dans des catégories qui nient la fluidité essentielle à la survie des ethnies. La non-ingérence, les lignes définitives du tracé des frontières, les droits historiques, la régulation des flux de migrants ne permettront plus aux populations de gérer leur territoire d'une manière "zomienne». Mais, les frontières resteront longtemps poreuses même s'il semble que l'on prenne conscience, paradoxalement au moment où la "globalisation " veut les supprimer, qu'elles sont au cœur du projet mondialiste du libéralisme. Il ne faut donc pas réfléchir l'anthropologie des frontières par le prisme des intérêts nationaux comme, par exemple, celui de l'accès aux ressources. On ne peut évidemment nier que les populations sont souvent chassées pour des raisons liées aux ressources mais la profondeur historique nous permet de comprendre que cette question [de l'accès aux ressources], bien que causant parfois des poussées de violence, finit toujours par s'arranger avec les populations « indigènes ». La reconnaissance internationale du problème fige les objectifs ethniques et nationaux dans des cases et les empêche de trouver des solutions naturelles. Car, il est un autre axiome à prendre en considération : aucun projet de développement ne peut se faire sans l'adhésion des groupes ethniques ou sociaux présents et, si des peuples sont menacés, ils n'en restent pas moins des substrats qui permettent aux nouveaux venus une adaptation et donc un syncrétisme. Les colonisateurs, les communistes, les "démocraties» ont essayé de classer les populations et de les "ranger» à l'intérieur de frontières définies - les montagnards du Vietnam ou aujourd'hui les nomades marins du sud de la Thaïlande. Il ressort des politiques étatiques des mouvements révélant des cultures que beaucoup de chercheurs, de développeurs et de politiques pensaient disparues. Le temps ethnique n'est pas notre temps et l'espace-frontière n'est pas un espace fixe.

9 La compétition pour les ressources, que mentionne Horstmann (2002) pour les sociétés étatiques et avant lui de nombreux auteurs américains pour les ethnies minoritaires (Hutterer et al. 1988), n'est qu'un avatar des relations nomades et sédentaires existant sur les marges. Mais, aujourd'hui, les chercheurs se fondent sur la vision libérale et même «commerciale» des anthropologues américains qui ne voyaient (en schématisant) dans l'acquisition des ressources qu'un moyen pour le commerce international de répondre au problème d'approvisionnement; les différentiations sociales et ethniques répondaient à ce besoin (certains Orang Asli -Homme des Origines - de Malaisieiii, par exemple, les Moken, les Mlabri...). Or, il s'agit bien plus d'un choix des populations, mais sans pour autant oublier les pressions facilitant la séparation d'avec un groupe plus important telles que les guerres, la surpopulation, le mouvement millénariste... Les besoins créés par les débuts de la mondialisation de la deuxième moitié $\mathrm{du} \mathrm{xx}^{\mathrm{e}}$ siècle mettaient à mal les choix des populations, par exemple, celui de lutter ou non. La non-violence est une condition de la survie des nomades d'Asie du Sud-Est; c'est aussi une raison de leur «fuite» (un avatar du nomadisme) c'est-à-dire de leur sauvegarde. Plutôt que de lutter, certains d'entre eux choisissent de partir. La non-accumulation est un autre moyen de survie car, en restant pauvres et en développant une culture sur cet axiome de l'impossible enrichissement qu'expliquent 
parfois les mythes (Ivanoff 2004), les nomades se protègent. La différence est bien dans la lutte. Si un peuple défend avec des revendications politiques et économiques son mode de vie par les armes, il retient l'attention et donc existe en tant qu' "État », créant un monde manichéen faisant fonctionner le fantasme international avec ses bons et ses méchants. Mais, les populations ainsi identifiées (les Karen par exemple) en payent le prix car, alors, elles doivent continuer à exister selon des critères définis ou rejetés par l'extérieur.

\section{Bref historique des mouvements aux frontières}

La mosaïque ethnique et sociale qui compose l'Asie du Sud-Est rend difficile l'abord des questions de migrations et des frontières, sans un éclairage de l'évolution historique.

11 - Le cadre général de cette réflexion est donc avant tout la relation complexe mais insécable entre les nomades et les sédentaires. Depuis la préhistoire, on observe des mouvements concomitants entre des marges mobiles et des centres sédentaires. L'archéologie sociale (Brun et Miroschedji 1998-1999) et certains anthropologues ont montré que les populations nomades des marges créaient un espace vital de rencontre dynamique interethnique, un laboratoire « d'essais socioculturels » et ethniques.

12 - Aujourd'hui, ces relations entre nomades et sédentaires s'inscrivent dans le cadre plus descriptif et moderne de centres et de périphéries. L'ethnicité ne disparaît pas complètement, mais les groupes ethnonationaux et les groupes ethniques se mêlent. Un autre type de mobilité entre le centre et la périphérie apparaît avec l'émergence des États qui tentent de rationaliser leurs relations.

13 - Viennent ensuiteles conflits de territoires qui entraînent des mouvements importants de populations. Il s'agit pour les États naissants et hégémoniques de transférer de la main-d'œuvre et des savoir-faire.

14 - Ces migrations de frontière se sont par la suite fluidifiées selon deux axes. Le premier s'arrangeait avec le concept des multivassalités (Thongchai Winichakul 2005) qui autorisaient plusieurs vassaux à être redevables à plusieurs suzerains. Un partage territorial d'un espace social à emboîtement donnait aux hommes, aux peuples et aux ethnies des frontières une certaine liberté de mouvement, non exempte de dangers et de razzias. Le deuxième axe était purement politique et militaire. Par exemple, l'expansion du Siam a provoqué des transferts de populations importants qui ont configuré les "cartes" migratoires contemporaines, que ce soit en Birmanie, au Cambodge, en Malaisie ou au Laos.

15 - Lors de la colonisation, les Occidentaux ont voulu empêcher ces fluidités migratoires en imposant des frontières et des lieux d'habitation fixes. Le Siam a dû alors gérer les mouvements des populations enfermées à l'intérieur de ses frontières et organiser la fluidité des migrations, qu'il s'agisse du déplacement de paysans du Nord-Est dans le Sud ou de l'enfermement des Malais dans la région de Patani.

16 Ces frontières dessinées à la règle, à la manière occidentale, ont séparé des populations mais ont ainsi établi les bases des futurs réseaux sur lesquels les trafics illégaux contemporains s'appuient. En effet, tant que la région frontalière participe à la construction nationale et n'a pas de velléités indépendantistes, le centre ferme les yeux sur cette fluidité remarquable, de part et d'autre de la frontière.Les réseaux transfrontaliers alors créés sont dirigés par les forces locales des «puissances 
sombres » (itthipan muet) comme les appellent les Thaïs. Ces puissances locales interdisent aujourd'hui la mise en œuvre de politiques modernes de gestion des flux migratoires. Elles sont plus réactives que les réglementations des gouvernements qui ne vivent que pour survivre. Les gouvernements ont une histoire et des liens transfrontaliers solides.

17 Aujourd'hui, l'armée et les mouvements paramilitaires régissent de fait les flux frontaliers et sont alliés à une pléthore d'officiels corrompus qui ont renforcé les trafics en les rentabilisant au maximum, au détriment bien sûr des victimes (Boutry et Ivanoff 2009).

18 Nous présupposons que la mise en place de projets de développement ou d'intégration nationale des peuples "périphériques " ou "rebelles à l'intégration» et désignés comme « archaïques» est impossible sans leur accord préalable. Ceux-ci construisent leurs propres outils de désenclavement sans avoir besoin de politiques centralisatrices qui reconfigurent leur ethnicité et leur espace social selon des critères exogènes.

19 Les migrations ont différentes caractéristiques mais redessinent les régions selon des stratégies de « colonisation adaptative » ou d'ethnorégionalismes. Par exemple, on peut avoir un mouvement de l'intérieur vers l'extérieur comme lorsque les Birmans ont colonisé le sud de la Thaïlande, ou bien, des mouvements intérieurs comme les déplacements des paysans du Nord-Est en Thaïlande. La loi du développement et du marché favorise cependant de plus en plus les Birmans qui valent moins chers que les paysans du Nord-Est. Les Isan retournent dans le nord-est de la Thaïlande, leur région d'origine, parce qu'ils sont, pour la plupart, remplacés par des travailleurs venus des pays voisins (Boutry et Ivanoff 2009). Ainsi, un espace social intermédiaire (ici le NordEst) peut aider son centre à coloniser d'autres marches:100 000 habitants de cette région avaient été déplacés dans le sud pour contenir la «menace » des musulmans; 15000 familles à qui l'on a donné des plantations pour récupérer la production.

20 La civilisation des marges passe aussi par l'appropriation des taxes et des circuits économiques. Ces derniers s'épanouissaient alors que des villages chinois officiellement inexistants et attachés aux plantations trafiquaient sans passer par Bangkok. On remarquera aussi que des villages entiers dans le district de Kaw Thaung, de l'autre côté de la frontière birmano-thaïlandaise, sont tout aussi administrativement inexistants. Il existe ainsi une population "inconnue», une main-d'œuvre docile servant les intérêts privés des développeurs locaux qui utilisent la dette, le chantage et l'esclavage pour développer les régions et les intégrer ensuite dans un régionalisme " acceptable ». Ces habitants migrants, non répertoriés mais que tout le monde connaît, développent les pays de leurs mains et au prix de leur liberté avec cependant le paradoxal avantage de la mobilité (surtout en Birmanie).

21 Pourquoi les autorités changeraient-elle ce système de mobilité qui enrichit tout le monde et qu'elles ne peuvent véritablement contrôler? Les pauvres vont tenter leur chance dans les pays riches qui profitent ainsi d'une main-d'œuvre bon marché. Les officiels et les filières gagnent de l'argent. Quelques règles permettent de montrer que l'on gère ces flux ; il existe bien des travailleurs réguliers mais, au vu de leur nombre et des échecs des tentatives de régularisation des travailleurs, on peut douter de la volonté réelle des gouvernements d'officialiser ces dynamiques. Ces migrations transfrontalières et illégales permettent la fluidité de l'ethnicité et offrent aux populations une possibilité de tisser des relations interethniques de manière « naturelle », c'est-à-dire sans intervention de l'État. Les pêcheurs birmans du sud de la 
Thaïlande sont un exemple de ce phénomène (Boutry et Ivanoff 2008). Il faut adapter la demande du marché à la réalité structurelle et culturelle des frontières, sinon aucune réglementation ne sera efficace. Comment gérer les déplacements des 4 millions de Birmans en Thaillande si l'on ne connait pas les routes, les intermédiaires, les relations qui lient les puezaiv birmans (courtiers) ou taukay ${ }^{v}$ aux hommes, si l'on ne connait pas les réseaux de distribution de l'information et les structures psychologiques présidant aux décisions de migrer? Mais la "bonne gouvernance » voudrait une transparence totale, ce qui est impossible. Les migrations illégales, comme la corruption, sont connues mais personne n'en parle à part quelques organisations non gouvernementales (ONG) impuissantes et quelques agences nationales qui agissent comme des boucliers pour préserver l'image des pays victimes d'attaques internationales trop violentes. Le cas des Rohingyas ${ }^{\mathrm{vi}}$ illustre bien ce propos. Quand la réalité explose ainsi derrière le fait divers, personne n'est préparé à l'affronter, à supporter la vue des millions de Birmans vendus. Une fois la situation révélée au grand public, comment la gérer? Accuser les Rohingyas de terrorisme pour justifier leur traitement? C'est fait. Mettre en avant le fait qu'ils représentent un problème interne à la Birmanie évite d'aborder la question d'un peuple sans identité et permet de détourner l'attention que l'on commence à porter aux millions de Birmans exploités dans les mines, les plantations et sur les bateaux. Les Rohingyas ont révélé la question birmane (on est passé officiellement de 1 à 2,5 millions de migrants) mais, en même temps, ils ont permis de la dissoudre dans des comités, des enquêtes fictives, de saisir l'ASEAN, bref, de cacher la réalité.

Les échecs d'intégration et de contrôle de l'État thaïlandais vis-à-vis des minorités vii laissent souvent aux populations des frontières le soin d'élaborer des stratégies d'entente ou de confrontation avec leurs voisins. Sans entrer dans les détails de la territorialisation des populations transfrontalières et des enjeux identitaires qu'elles posent aux États, disons ici que leurs mouvements transfrontaliers traditionnels préfigurent les mouvements migratoires, leurs routes et leurs formes.

Les nouveaux migrants, poussés par des facteurs politiques et économiques, empruntent, à un moment ou un autre, les mêmes routes que les nomades transfrontaliers pour, ensuite, étendre leurs réseaux tout en dominant peu à peu ces mêmes populations transfrontalières. Nous assistons alors à la première colonisation adaptative, celle qui assimile les premiers habitants des zones de passage et jette une passerelle entre deux pays. Entre le laxisme ou l'autoritarisme des gouvernants des pays dont elles relèvent, les populations des frontières construisent leurs nouveaux territoires d'expansion spatiale et d'expression sociale. Comment et avec quels moyens culturels ou ethniques y parviennent-elles? Ces nouvelles relations interethniques liées aux migrations posent de nouvelles questions à l'anthropologie. Existe-t-il une idéologie propre aux ethnies des frontières qui leur permettrait une adaptation plus rapide et plus constante de leur identité ? Comment les migrants transfrontaliers utilisent-ils les mêmes méthodes pour reconstruire des espaces sociaux au-delà de la frontière et, in fine, en se mélangeant aux populations autochtones, comment déterminent-ils les différences sociales ou ethniques ? En effet, on constate depuis fort longtemps des phénomènes de segmentations ethniques et/ou sociales.

Reprenons ici l'exemple des Moken: proto-malais issus d'une matrice malaise dominant un vaste archipel (plusieurs centaines d'îles étalées sur deux pays), ils sont devenus une ethnie à part entière ; en dépit des conditions historiques (esclavage, islam...) qui les ont de facto et par choix coupés du monde malais, de l'islam et des Thaïs, 
ils n'ont pas une ethnicité déterminée puisque les mouvements actuels de populations les transforment - comme les Mlabri, liés à l'origine aux Laos, et qui ne sont pas en voie de disparition comme on l'a longtemps cru. Les Mlabri du Laos et de la Thaïlande sont également des segments de la population laotienne qui se sont séparés de leur groupe dominant pour définir un nouveau groupe en s'alliant sur les bases d'un échange inégal avec d'autres populations de dimension « intermédiaire " tels les Hmong. De même, les Birmans venus coloniser les eaux du sud du pays ont transformé leurs marqueurs identitaires en s'associant avec des nomades. Et les Malais du sud de la Thaillande ont eux-mêmes créé un espace social intermédiaire qui fait d'eux une population ni malaise ni thaïlandaise. Les migrations ont donc une influence très importante sur les constructions identitaires contemporaines exacerbées par les possibilités offertes par la frontière. Nous pensons (Boutry et Ivanoff 2008) que les anthropologues sont passés trop rapidement sur l'ethnicité de «base » pour reprendre une terminologie obsolète des ethnopsychiatres. Il existe selon nous une limite à l'interaction et à l'acceptation de transformations. Une perméabilité structurelle à la société est nécessaire à l'adaptation mais elle a des limites car, au-delà d'un certain point, c'est l'ethnie ou le groupe qui disparaît. Nous en avons un exemple avec les interactions entre Moken et Birmans, Thaïlandais et Moken. Même si les Moken diminuent en nombre à cause des relations interethniques avec les Birmans, la population qui résultera des mariages « mixtes » ou de «l'exogamie culturelle» (Boutry 2007) les aura sauvés d'une disparition ou d'une transformation radicale certaine - due à la raréfactions des ressources, aux maladies des sédentaires, à la mobilité de plus en plus difficile-, et la société moken en ressortira renforcée car elle aura trouvé un moyen de s'associer, le temps nécessaire à sa recomposition, si recomposition il y a. L'association avec les Birmans permet l'attentisme et le mouvement millénariste qui se développe, laisse penser que les Moken n'ont pas dit leur dernier mot en Birmanieviii. Au contraire, la non-interaction avec les Thaïlandais dans les parcs nationaux a permis d'augmenter la démographie. Ceci prouve deux choses : 1) la résilience de la société qui resurgit (à travers les rituels notamment) et 2) la nette chute de la mortalité infantile, cause d'un regain démographique.

L'étude des frontières permet de mieux comprendre les volontés idéologiques ou le pragmatisme des États face aux politiques internationales et aux fantasmes nationaux de contrôle. Quelles sont les conséquences des politiques et comportements des nations pour les groupes concernés : adaptation, disparition, renouveau, etc.? Les réponses culturelles apportées à ces nouveaux défis sont beaucoup plus subtiles que ne le pensent développeurs et économistes. En effet, en s'appuyant sur des traditions syncrétiques, ces groupes s'adaptent dans un creuset ethnique préparé par le socle socioéconomique et religieux préexistant. Cela provoque des nouvelles formes de mobilité transfrontalière (éclatements en segments, regroupements avec d'autres parents éloignés...) et socioculturelle (adaptations techniques, apprentissage des langues...).

Mais, souvent, la question de la dénomination de ces populations se pose, une fois la frontière passée. Doit-on les appeler des immigrés, des réfugiés ou bien des illégaux ? Ces classifications nécessaires aux Occidentaux brouillent la piste de l'ethnicité. Ces catégories inventées par les organismes internationaux et étatiques déterminent l'avenir et la cohésion de ces peuples utilisés lors des marchandages entre pays voisins. Les flux sont ainsi sémantiquement gérés ou non - le statut des réfugiés en Thaïlande, par exemple, change selon les gouvernements et les intérêts de chacun - mais, selon la 
réalité des chiffres, ces catégories facilitent l'entrée dans l'illégalité pour tous ceux qui n'y trouvent pas leur place. Il n'existe pas d'homogénéité dans les réponses apportées par les populations des frontières aux contradictions posées par le monde contemporain; par contre, elles révèlent des «fondamentaux ethniques», une ethnicité qui leur permet de s'adapter, de composer parfois, de disparaître aussi. Car, souvent, un "choc» historique crée des ethnies soumises ainsi à leurs dynamiques internes et aux pressions extérieures. Ces chocs, créateurs d'ethnicité (Godelier 2009), renvoient ensuite à la question du choix culturel ethnique (choix de civilisation dirions-nous aujourd'hui) que Benjamin (1988) a bien mis en avant.

Nous pouvons donc analyser en temps réel les retours culturels, les réponses des peuples frontaliers, leurs techniques, leurs innovations, et leurs capacités syncrétiques ou encore leurs redéploiements identitaires. Le territoire de ces marges, très souvent fantasmé par le centre (peur du vide, de la sécurité, du sauvage...), est en fait redéfini dans son expression et sa conception. Les territoires frontaliers se reconstruisent, s'interpénètrent et, surtout, utilisent la dynamique centre/périphérie pour concevoir de nouveaux modes de représentation de l'espace. Ceux-ci sont exprimés par les dynamiques interethniques et les nouveaux espaces d'expression culturelle, mais aussi par l'histoire de ces peuples, jalonnée d'expériences de confrontations culturelles et qui leur permet de concevoir des stratégies ethniques pour s'adapter.

Le nouveau cadre international et les contractions nationales à propos des frontières (repli identitaire, imaginaire du territoire du vide, association de l'ethnicité et de territoire national...) offrent aux peuples des frontières des opportunités de se positionner en tant que culture particulière, de jouer la carte de la segmentation ethnique, de l'association entre "cousins » éloignés, de se déplacer, d'imaginer de nouveaux espaces. C'est l'anthropologie des frontières. Ces peuples offrent donc un panel intéressant de révélations de latences culturelles redéfinissant le "périmètre " de chaque groupe. Plus le centre avance dans sa volonté de contrôle et se renforce à l'aide de l'idéologie globalisante internationale, plus les ethnies se reconfigurent.

\section{Des voisins difficiles : migrations et catégorisations des " autres » en Thaïlande}

En Thaïlande, les données quantitatives sur les migrations sont évidemment difficiles à obtenir. La réalité des chiffres officiels est plus que douteuse, celle des ONG est plus réaliste mais reste fonction de leurs intérêts. Nous prendrons ici un exemple chiffré concernant le nombre de travailleurs enregistrés en Thaïlande (Source : ministère du Travail). 
Enregistrement des travailleurs migrants 2002-2005

\begin{tabular}{|c|c|c|c|c|}
\hline Secteur & Birmans & Cambodgiens & Laotiens & Toutes nationalités \\
\hline \multicolumn{5}{|l|}{ Pêche et à-côtés } \\
\hline 2002 & 74775 & 19407 & 1412 & $\$ 5594$ \\
\hline 2000 & 4644 & 8659 & 761 & 56862 \\
\hline 2004 & 96101 & 27540 & 3647 & 127288 \\
\hline 2005 & 85058 & 16633 & 2785 & 104474 \\
\hline \multicolumn{5}{|c|}{ Travail domestiqu } \\
\hline 2002 & 50043 & 3255 & 12068 & 65361 \\
\hline 2008 & 40909 & 2511 & 9265 & $526 \% 5$ \\
\hline 2004 & 88319 & 8746 & 31499 & 128514 \\
\hline 2005 & 67789 & 9242 & 27306 & 105306 \\
\hline \multicolumn{5}{|c|}{ Travail ousurier } \\
\hline 2002 & 68,417 & 3255 & 10331 & 91618 \\
\hline 2000 & 46,634 & 3683 & 4897 & 52756 \\
\hline 2004 & na & $n a$ & na & $\mathrm{na}$ \\
\hline 2005 & na & na & na & na \\
\hline \multicolumn{5}{|c|}{ Tous secteurs } \\
\hline 2002 & 340029 & 5989 & 32492 & 409339 \\
\hline 2000 & 247791 & 1964 & 21314 & 288790 \\
\hline 2004 & 610106 & 104789 & 99352 & 814247 \\
\hline 2005 & 589416 & 75804 & 90073 & 705296 \\
\hline
\end{tabular}

exploitation situations of migrant workers in Thailand, 2007 : 2 (Asian Research Center for Migration, Institute of Asian Studies, Chulalongkorn University).

Les auteurs reconnaissent eux-mêmes qu'il faut doubler les chiffres du tableau cidessus,car ils n'incluent pas les travailleurs et les personnes qui leur sont dépendantes (femmes et enfants). De 700 000, on passe à 1,4 million. Officiellement, on sait que deux fois plus ( 2,8 millions) de travailleurs étrangers ne sont pas enregistrés. Si l'on tient compte des calculs effectués à partir de données recueillies lors d'une enquête parue sur la filière pêche (Boutry et Ivanoff 2009), on peut multiplier les chiffres par deux pour l'ensemble des travailleurs et avancer une estimation réaliste de 5 millions de personnes.

31 Avant de parler du cas particulier de Ranong pour les Birmans travaillant dans la filière pêche, il faut savoir que le ratio d'un étranger pour trois travailleurs thaïlandais dans les 13 provinces frontalières, passe à 1 pour 9 à Ranongix. Ainsi, le nombre officiel de travailleurs birmans en Thaïlande passe à 500 000, auquel s'ajoutent 500000 autres non-officiels mais reconnus. Mais, la réalité est différente et le scandale des Rohingyas rejetés à la mer a obligé les autorités à donner des statistiques plus réalistes. Les chiffres sont ainsi passés, dans le courant de l'année 2008, de 1 à 1,5 puis à 2 millions, et enfin, à 2,5 millions. Les compteurs semblent être bloqués là, il existe une limite à la transparence. Il y a vraisemblablement, selon nos estimations, 4 millions de Birmans en Thaïlande.

\section{Structuration des filières migratoires}

32 Nous décrivons deux réalités qui se superposent et fonctionnent ensemble, celle d'une Thaillande moderne et intégrée et celle d'une Thaillande aux archaïsmes puissants dont les ethnorégionalismes sont la colonne vertébrale et dont les projets de développement 
sont un moyen de conserver le pouvoir. Pourtant, malgré les projets, les habitants du Nord-Est se considèrent comme proches des Laotiens, voire même comme des Laotiens, tout en étant des citoyens thaillandais. De même, les projets de développement du Sud, qui apportent des millions de dollars au nom d'un sous-développement imaginé des marges et qui servent à l'armée et aux porte-parole locaux, n'ont pas réussi à éradiquer la double identité thaïlandaise et malaise. Le but, dans un premier temps, est de fixer les populations avant leur ouverture au monde libéral par l'implantation de projets de développement censés apporter un soutien de la population à la nation et aux élites (Ferguson 1994).

Ceci fait apparaître un nouveau paradoxe : les pays frontaliers développent l'économie des marges, notamment avec des infrastructures, pour faciliter la mobilité et l'échange, mais aussi pour fixer les populations "pauvres ». Tous les gouvernements pensent que le développement permettra le contrôle des migrations et stoppera les velléités séparatistes et les afflux massifs de travailleurs illégaux. On arrive ainsi à des contradictions évidentes. Lors d'une visite dans le Sud rebelle de la Thaïlande, le Premier ministre malaysien affirma que les habitants musulmans, surtout, devaient loyauté à la Thaïlande et au roi. Mais, il a également déclaré que les liens économiques entre les deux pays allaient être renforcés; le pont qui sépare Sungai Kolok de la Malaysia, et par où passe le plus grand nombre de migrants et de marchandises, est baptisé le «pont de l'amitié» (entre les gouvernements qui reconnaissent officiellement ne pas intervenir dans les affaires de l'autre, ce qui facilite l'illégalité). En même temps, la suppression annoncée de la double nationalité risque de favoriser l'illégalité de dizaines de milliers de privilégiés qui ne cesseront de faire des allers et retours mais qui seront plus susceptibles de tomber dans les mains des filières plus ou moins maffieuses ou manipulées par le pouvoir local.

Pourquoi cette volonté de fixer les populations sur des marges jugées incertaines? Si les "minorités ethniques" en Thaillande posent un "problème » aux marges de la nation (fantasme du contrôle de territoire que les ethnies menacent), qu'en est-il des gens du Nord-Est et des Malais du Sud de la Thaïlande? Ces derniers, ethniquement différents des Thaïs, ne sont pas une minorité ethnique ni même nationale: la Thaïlande impose un creuset collectif unique fondé sur la culture et non pas sur la « race ». Mais, ces deux groupes n'en demeurent pas moins très fortement différenciés et reconnus comme tels par le centre imaginé des Thaïlandais. Qu'est-ce qui les différencie d'un groupe ethnique? Ils vivent aux frontières, ont leur langue et leurs traditions, sont rattachés à des groupes « frères » de l'autre côté de la frontière ce qui permet des échanges, ils s'adaptent et construisent des objectifs de solidarité locale pour survivre en tant que groupe. Il faut donc reconsidérer les frontières coloniales et leurs conceptions pour mieux comprendre, d'une part les flux entre pays et, d'autre part, la volonté paradoxale de fixer les peuples aux frontières.

Avant la colonisation, chaque morceau de territoire déclaré indépendant par les puissances locales pouvait lors du démarquage des frontières être attaché à une autre portion de territoire. Les limites des frontières étaient discontinues et les royaumes morcellés territorialement ce qui facilitait le passage et la mobilité; aujourd'hui encore, les candidats à la migration clandestine utilisent ces passages traditionnels. Autrefois, une simple maison et un gardien ne représentaient rien pour les habitants des deux côtés qui pouvaient s'installer là où ils le désiraient. 
Même dans le cas de voisins ennemis des arrangements étaient possibles. Ces relations commerciales, sociales ou de parenté perdurent aujourd'hui sous d'autres formes et maintiennent les régions à l'intérieur de la nation thaïlandaise contemporaine. Généralement, un corridor, ou zone tampon, couvrant les limites de territoires ennemis, permettait aux habitants de passer d'un côté ou de l'autre sans dépasser la limite où l'autorité de chaque royaume s'exerçait. La souveraineté et la frontière n'étaient pas reliées. Un royaume pouvait définir son propre territoire et délimiter sa frontière sans l'accord de l'autre partie. Quand, à la fin du règne de Rama III, la question de la frontière entre Tenasserim et le Siam a été posée, le Siam a déclaré à la fin de la guerre anglo-birmane que "les frontières du Tenasserim ont été étendues pour le rejoindre ». L'ouverture des frontières signifie des relations de bon voisinage à l'époque moderne alors, qu'autrefois, il y avait pour marquer ces bonnes relations un " chemin d'or, d'argent » emprunté par les uns et les autres. Les relations de pouvoir en Asie du Sud-Est sont connues sous le nom de mandala et, comme Wolters (1999: 16-17), nous pourrions proposer la définition suivante :

[The] mandala represented a particular and often unstable political situation in a vaguely definable geographical area without fixed boundaries and were smaller centers tended to look in all directions for security. Mandalas would expand and contract in concertina-like fashion. Each one contained several tributary rulers, some of whom would repudiate their vassal status when the opportunity arose and try to build up their own networks of vassals.

On voit donc la complexité des relations entre États voisins, suzerains et vassaux, et celle d'un réseau à multiples dominations. Ces situations sont encore courantes aujourd'hui. Il s'agit toujours de dons et de contre-dons; c'est pourquoi Thongchai Winichakul a raison de mentionner (2005: 87) le don de Marcel Mauss pour ceux qui essaieraient de comprendre les relations interethniques et interétatiques. Aujourd'hui encore, le sud de la Thaïlande perpétue par exemple ce système de relations de multivassalités et de multisuzerainetés, souvent fondées sur l'exploitation du territoire par des ethnies différentes (Ferrari 2008). La relation entre le don et le contre-don, en fait l'acceptation de multiples vassalités aux pouvoirs inégaux et parfois même théoriques - mais qui entraînent un certain respect et une tolérance dans l'accession aux ressources - est à la base des relations entre les personnes (paternalisme, népotisme, omniprésence du taukay...), les États (Bunga Mas, les tributs symboliques ou non...), entre les génies ou les divinités tutélaires d'un peuple et le génie du lieu. Tous ces rapports à emboîtement (Condominas 1978) sont entretenus par l'intermédiaire d'un syncrétisme rituel puissant qui influe sur la mobilité. Les nomades resteront nomades puisqu'ils ont réussi à intégrer les marqueurs essentiels des dominants sans se faire absorber. Les Chinois continuent à chercher fortune et à travailler en s'intégrant dans tous les interstices possibles (y compris dans le monde musulman), déplacent des villages entiers à travers la région, et même, font venir de Chine des travailleurs en tout illégalité. Les Malais du sud de la Thaillande, suivant en cela leurs traditions migratoires et de domination, continuent leur marche sur les fronts pionniers et remplacent les gens du nord-est sur la frontière. Ils recolonisent à leur profit les marges de la Thailande.

L'endocolonialisme siamois, puis thaïlandais, a donc créé des espaces sociaux intermédiaires plus ou moins velléitaires quant à la défense de leur culture et de leur appartenance à un bloc, et à l'intérieur desquels, les segments lao et malais coupés de leur matrice ont développé des caractéristiques culturelles et des traditions anciennes 
qui disparaissent ailleurs. Des segments entiers de populations se sont séparés de leur matrice, ont construit de nouveaux repères, de nouvelles routes migratoires, et ont tissé de nouveau liens. Ainsi, une nouvelle mobilité s'est développée avec l'éclatement de populations par la colonisation.

Il faut aussi mentionner les reconfigurations des mobilités depuis la colonisation occidentale et l'endocolonialisme siamois. Les Malais du Sud de la Thaïlande émigrent vers la Malaysia pourvoyeuse d'emplois pour des «frères en religion » opprimés. Cette unité ethnique et cette solidarité ne peuvent cacher le fait que les Malais de Patani ont développé un espace particulier et qu'ils jouent entre les antagonismes thaïlandais et malaysiens. À l'inverse, les Laotiens viennent en pays isan pour travailler et ces mêmes Isan partent à travers tout le pays pour chercher des opportunités de travail. Il y a un effet domino qui a suivi la démarcation des frontières. Mais, ces mouvements changent et le Cambodge et la Birmanie deviennent désormais les pourvoyeurs principaux de main-d'œuvre. Les gens du Nord-Est ont ainsi perdu une partie de leurs possibilités d'emplois tout en se développant - relativement selon que l'on compare leur niveau de vie à celui des habitants de Bangkok ou des Cambodgiens par exemple. Ces espaces sociaux intermédiaires fonctionnent avec le centre et les autres pays limitrophes, mais ne jouent pas l'un contre les autres. En fait, ils deviennent les gardiens des traditions et d'un fonctionnement traditionnel d'interrelations sociales et ethniques. Les ethnorégionalismes sont les mémoires des pays de l'ASEAN qui ne survivraient pas sans eux, en tant qu'entité. Et, surtout, ils ont construit et conservé des réseaux, des relations d'entraide et d'exploitation qui permettent aux migrations de s'internationaliser. Il y a d'abord une migration « naturelle » qui fait bouger le segment laotien, khmer ou birman en Thaillande, parmi les proches (khmerophones de Thaïlande pour les Khmers, Isan pour les Laotiens...) qui habitent déjà la région pour $\mathrm{y}$ travailler. Il y a aussi les Malais qui vont en Malaysia pour des raisons aussi bien économiques que religieuses. Il existe donc une plateforme d'échanges qui permet le premier pas vers l'internationalisation des migrations que nous constatons avec les Birmans.

\section{Nationalisme, histoire et frontière}

L'économique et le politique se brouillent quand on étudie la notion de fronts pionniers c'est-à-dire la volonté de recouvrir ses frontières avec le peuple dominant (ou un de ses segments, le Nord-Est en Thaïlande, par exemple). Les frontières coloniales imposées ont entraîné des déplacements. Il s'agit de les conquérir. Par exemple, lors du conflit franco-siamois concernant les habitants de la région du Mékong, il a fallu décider qui appartenait à qui, puisque le système à emboîtement et les multivassalités n'était pas pris en compte. Un accord fut trouvé, mais qui entraînait le choix d'une résidence décidant de l'appartenance de la personne à tel ou tel pays. On a ainsi freiné la mobilité et rendu les migrations illégales car cette obligation de sédentarité allait à l'encontre d'un monde qui se divisait.

41 Ces coupures arbitraires ont entraîné la création de nouveaux flux (réfugiés politiques, travailleurs...) et, aujourd'hui, les pays concernés préfèrent laisser les réseaux traditionnels gérer les flux migratoires transfrontaliers plutôt que de tenter des négociations bilatérales ou multilatérales impossibles à appliquer. Car, les coupures sont encore des blessures fragiles que le nationalisme récupère très vite. On l'a encore 
vu à Phra Vihar où la dernière étape logique serait un déplacement de populations pour interdire aux voisins de coloniser ces $4 \mathrm{~km}^{2}$. L'armée devient paradoxalement le porteparole de la volonté de contrôle et de non-débordement.

La querelle actuelle entre le Cambodge et la Thaïlande, suscitée par l'inscription au patrimoine mondial de Phra Vihar et renforcée par la visite de Thaksin à qui le Premier ministre cambodgien a offert un poste officiel de conseiller économique, n'est que la continuation de la volonté d'un vassal de se libérer des contraintes d'un suzerain exigeant économiquement - on rappelle dans les journaux aujourd'hui en Thaïlande le statut d'ancien vassal lorsqu'on parle du Cambodge et au Cambodge on parle de l'agresseur siamois. Mais, au-delà même des relations bilatérales, on voit tous les jours en Thaïlande les " archaïsmes » historiques revenir et empêcher l'ASEAN d'entrer dans le club des décideurs de la planète. Il y a toujours un "quelque chose » entre certains pays qui empêche de dépasser le stade du consensus, c'est-à-dire du principe de ne pas faire " perdre la face » à un autre. L'histoire est trop chargée et les symboles trop forts pour ne pas réveiller des relations qui n'ont fondamentalement pas changé. Mais, si ces querelles politiques ne facilitent pas la mobilité, elles ne la freinent pas non plus. Le déplacement de groupes de travailleurs ou de réfugiés politiques et leur utilisation à des fins politiques demeurent. On parle de bloquer la construction de la route Surin en Thaïlande-Siam Reap au Cambodge qui, précise la Thaillande, apporterait beaucoup d'argent au Cambodge. Y-aurait-il autre chose que l'argent en jeu ? Alors, se demandent les politiques et les développeurs ou stratèges des grands argentiers internationaux, le Cambodge joue-t-il avec le feu? L'histoire ne lui a pas permis de régler ses comptes avec la Thaillande; on peut aussi imaginer que, derrière cela, d'autres marchandages économiques (le gaz dans le Golfe du Siam par exemple) ou l'apparition de nouvelles alliances - la Chine qui vient aider le Cambodge en prêtant de l'argent - sont dans la balance. Et, derrière la rhétorique nationaliste, la réalité demeure. Le Cambodge vient d'accorder une licence d'exploitation de ses eaux à 1000 chalutiers thaillandais. Avec cet accord, des dizaines de milliers de Cambodgiens se déplaceront en Thaillande.

\section{Les ethnorégionalismes comme préalables aux grandes migrations}

43 La politique contemporaine visant à créer des "corridors » ou des «triangles » de développement représente toute une géométrie variable de l'expansion volontariste du marché mais révèle un problème: la porosité des frontières et le fonctionnement traditionnel des passages, des accords, des réseaux et des trafics. Cette volonté libérale met surtout au jour des identités fortes aux frontières et des mouvements de colonisation interne génératrice de conflits.

En Asie du Sud-Est, l'ethnorégionalisme est le résultat de l'expression d'une identité révélée par un choc historique (ici l'imposition des frontières et la colonisation siamoise) avec ce que provoque un tel choix au niveau de l'identité: Siamois, Lao, Laotien, Thaïlandais, Thaï, Khon Isan ou Isan ${ }^{\mathrm{x}}$ pour le Nord-Est ; Thaïlandais, Khaèk ${ }^{\mathrm{xi}}$, Thai-Islam, Oré Nayu ${ }^{\mathrm{xii}} . .$. pour le Sud-Est. La langue, la nourriture, les habits, les traditions, la tentation communiste, tout porte ces deux régions à développer une volonté séparatiste politique ou culturelle. Se créent alors des mouvements de populations à l'intérieur d'un nouvel espace social; celui-ci, intégré à un ensemble national, devient à son tour pourvoyeur de migrants intérieurs. Mais, il n'y a pas de conflit «ouvert» sur cette question de mobilité et le phénomène de multiethnicité 
(Horstmann 2002) montre que la cohabitation de deux systèmes peut s'enrichir (Boutry et Ivanoff 2009). Nous parlons ici de millions de personnes et donc d'autant de segmentations possibles en fonction du lieu, de la frontière, du système d'échange local fondé sur des rapports de domination ethnique et des multivassalités anciennes (Thongchai Winichakul 2005). Ces segments, ou " corpus identitaires » dangereusement et potentiellement séparatistes, se rattachent à la capitale à travers l'invention d'un centre et d'une idéologie et parce que la Thaïlande a créé une région vivant sur des constructions socioculturelles qui ne doivent rien au centre mais ne s'y opposent pas non plus, en laissant s'exprimer des relations traditionnelles qui profitaient aux puissants de la région et aux politiciens de Bangkok.

Une des questions essentielles est de savoir comment l'articulation s'effectue entre une volonté régionale (au niveau de plusieurs pays, Union européenne et ASEAN, par exemple) et une résistance ethnonationale. Car nous parlons ici d'ethnorégionalismes à l'intérieur d'un cadre imposé par des frontières récentes. Mais il est certain que si l'Union européenne favorise l'émergence de pôles identitaires et économique (Catalogne par exemple), l'Asie du Sud-Est est différente ; elle essaie de construire un modèle qui endigue les ethnorégionalismes, des ponts (au sens propre et figuré) qui les enjambent et des corridors qui les intègrent. Les corridors (Moulmein, Khon Kaen...) ont été créés pour essayer de casser cette dynamique ethnorégionaliste des nations et globaliser le flux afin que disparaissent les développements inégaux. Mais, quand bien même les villes du nord-est bénéficieraient d'une arrivée de capitaux qui faciliterait l'arrivée de migrants, la fracture entre les Isan et les autres n'en serait pas moins vraie. On peut même penser que ces développements urbains (fournisseurs d'emploi donc freinant l'exode rural...) ne feront que reproduire à l'échelle locale les déséquilibres $\mathrm{du}$ pays, bombes sociales à retardement comme les mouvements des chemises rouges et jaunes l'ont montré (Ferrari et. al. 2010).

\title{
Développement, mobilité et identité
}

\author{
La mobilité surmoderne s'exprime dans les mouvements de populations \\ (migrations, tourisme, mobilité professionnelle), dans la communication générale \\ instantanée et dans la circulation des produits, des images et des informations. Elle \\ correspond au paradoxe d'un monde où l'on peut théoriquement tout faire sans \\ bouger et où l'on bouge pourtant. \\ Cette mobilité surmoderne correspond à un certain nombre de valeurs \\ (déterritorialisation et individualisme) [...] Mais, notre monde est plein de contre- \\ exemples: des exemples de sédentarité forcée, d'une part, des exemples de \\ territorialité revendiquée, d'autre part. Notre monde est plein d'«abcès de \\ fixation » territoriaux et idéologiques. (Augé 2009 : 7-8).
}

La mobilité des hommes est au centre du débat sur la liberté que permettraient les communications modernes mais aussi sur les résiliences et les archaïsmes qui continuent à creuser le déséquilibre entre les pays riches et les pays pauvres. Cette tendance de la recherche en sciences sociales à voir un monde supranational (monde de l'information par exemple) et donc la mobilité factice qu'offre le monde contemporain, crée des obligations « indigénistes » chez certains anthropologues qui pensent possible d'aider les populations à retrouver à travers cette "mobilité surmoderne" une ethnicité perdue (Karen et Hmong particulièrement, populations éclatées sur plusieurs pays et parfois même vivant dans le monde occidental). La disparition des frontières, les mouvements indigénistes et les moyens de communication permettraient alors de 
retrouver un «centre » ethnique perdu. On veut fondre deux notions, le nomadisme classique avec « le sens du territoire du temps et du retour » (Augé 2009: 7), pour n'en faire qu'une en accord avec le communautarisme américain : la version indigéniste des ethnologues contemporains. Ainsi font les déconstructionnistes français qui refusent les notions d'ethnies (Ivanoff 2003). Les ethnologues évitent par exemple de parler d'ethnicité particulière en prenant en considération plusieurs populations en même temps, d'autres pensent que les mouvements religieux (les missionnaires chez les Karen par exemple) créent une nouvelle ethnicité qui n'était qu'un terme mal défini (car n'entrant pas dans leur logique communautaire).

Si Marc Augé est au cœur du débat sur la mobilité, pour le nomadisme contemporain il faut relativiser la notion de l'homme libre et nomade, le riche donc qui détruit les archaïsmes culturels pour la plus grande mobilité de la minorité libérale... Marc Augé se réfère à un modèle libéral où les technologies de la communication effaceraient les obstacles liés à l'espace et au temps.

D'un autre côté, ceux qui rêvent d'une société humanité et considèrent que la planète est leur patrie ne peuvent ignorer ni la force des replis communautaires, nationaux, ethniques ou autres, qui veulent rebâtir des frontières, ni l'expansionnisme des prosélytismes religieux, qui rêvent de conquérir la planète en bousculant toutes les frontières.

Dans le monde "surmoderne ", soumis à la triple accélération des connaissances, des technologies et du marché, l'écart est chaque jour plus grand entre la représentation d'une globalité sans frontières qui permettrait aux biens, aux hommes, aux images et aux messages de circuler sans limitation et la réalité d'une planète divisée, fragmentée, où les divisions déniées par l'idéologie du système se retrouvent au cœur même de ce système. (Augé 2009 : 13-14)

L'Institut de recherche sur l'Asie du Sud-Est a lancé un programme de recherche sur la mobilité et les frontières ${ }^{\mathrm{xii}}$ et prépare un ouvrage, Peuple des frontières ${ }^{\mathrm{xiv}}$, qui s'inscrit dans un débat plus général sur la modernité, la mobilité, l'ethnologie de celle-ci et le déplacement de concepts (mobilité, nomadisme) ou l'affirmation d'autres comme étant réglés (ethnicité, communauté). Cependant, le monde des ethnies continue de tourner et les conséquences des politiques continuent à jouer sur l'ethnicité et la mobilité le rôle des empires et des thalassocraties d'autrefois.

Le nouveau rapport de forces fondé sur des frontières pratiquement poreuses mais fermées officiellement est né de la mainmise libérale sur les périphéries, une nouvelle manière de faire des bénéfices en détruisant les barrières nationales et protectionnistes. Personne ne veut s'aliéner les populations n'ayant aucun intérêt à se montrer trop infidèles envers un centre qui, s'il se raidit parfois, laisse agir les us et coutumes locales, notamment dans la formation des élites et des nouveaux riches. Il ne s'agit pas du tout d'États faibles, incapables de contrôler les périphéries comme certains auteurs décrivent les nations sud-est asiatiques. Certains historiens occidentaux (Reynolds 2002; Keyes 2008; Brown 1994, etc.) n'ont pas compris le pragmatisme et la continuité historique qui permettent de gérer des situations contradictoires en apparence, mais qui ne font que reprendre les données anciennes de multivassalités (Thongchai Winichakul 1994) en les adaptant au discours libéral moderne. Ces historiens et anthropologues ne comprennent la construction de l'Étatnation que dans sa dissolution dans une économie-monde libératrice des archaïsmes un discours né dans les think tanks de l'époque Blair/Clinton, qui propose de libérer les individus de leurs entraves ethniques, culturelles et donc, surtout, corporatistes et syndicales, pour leur offrir la véritable liberté, celle d'être soumis aux forces du marché 
régulateur. Celui-ci bien évidemment les broierait dans une flexibilité insupportable du point de vue psychologique mais, en l'absence de l'idéologie communiste (bien que celle-ci ait voulu également «libérer " les entraves ethniques), qui peut s'opposer à cette idéologie? En Thaïlande, pour survivre, les gouvernements doivent payer ceux qui les ont élus. Cela se fait à travers l'attribution de crédits, de développements d'infrastructures, de projets. Mais, cela se fait également à travers les budgets alloués à l'armée pour " contenir » le sud. Le gouvernement thaïlandais n'a en fait que très peu de marge de manœuvre sur ces frontières, ce qui facilite évidemment le trafic et la corruption.

50 Le pays continue cependant de donner de l'argent, de construire des infrastructures, de se développer. Pourtant, bien que la situation, depuis un siècle, ne se dégrade pas outre mesure (sauf, depuis 2001, à la suite de la politique de Thaksin qui a cru pouvoir régler le problème «Sud» de force), aucun enseignement n'a été tiré de l'échec politique de cette stratégie de développement pour lutter contre une tendance au séparatisme officiellement liée au sous-développement. Et, il est à craindre que le gouvernement thaillandais ne continue cette politique dispendieuse et inutile, pour essayer de régler la question de la division entre la classe aisée et urbaine et celle des paysans laissés pour compte qui ont fait la une des journaux au printemps 2010. Le Sud-Est est riche et sa diversité n'a jamais empêché les gens de s'enrichir, mais les réseaux d'entraide et d'échange sont complexes dans une péninsule où se rencontrent les Asies du Sud-Est insulaire et continentale. La région n'a pas attendu les crédits du gouvernement ou les politiques de développement pour s'enrichir à son rythme. En voulant intégrer nationalement ou, au contraire, régionalement, la Thailande a, en fait, renforcé une identité locale très forte. Le nouveau gouvernement démocrate d'Abhisit Vejjajiva n'agit pas autrement que ses prédécesseurs : visiter, écouter et payer sans comprendre. La seule différence réside dans le fait que les gens du Sud demandent à être traités différemment au niveau économique (c'est le nouveau moto du gouvernement, la vision bottom up et non plus top down, imposée par la volonté de décentraliser). Bien entendu, de telles idées sont lancées par les leaders économiques, autrement dit les profiteurs de la corruption généralisée. Quand Surachai, le président de la chambre de commerce du sud de la Thaillande, veut promouvoir la ville de Hat Yai comme un centre de plaisir et le reste du sud comme un centre de production de produits halal, il découpe la frontière, et donc, la mobilité des gens qui vivent de chaque côté selon un schéma socioéconomique : une maison close à la grandeur de la ville (Hat Yai, ville frontière faisant partie de la "vraie» Thaillande bouddhiste et libérale), ville ouverte pour les Malaysiens d'origine chinoise le plus souvent d'un côté et, de l'autre, une région plus musulmane et plus pure, ouverte au commerce. Ce genre de projet va contribuer à accentuer la séparation culturelle des deux régions et entraînera des migrations dans les deux sens. Les deux nouveaux pôles cassent la dynamique régionale sud tout en faisant gagner de l'argent aux puissants locaux. Voilà un moyen de marginaliser un peu plus la région «halal " par rapport à celle du sud et de faire payer le gouvernement pour la fidélité de ses hommes d'affaires influents.

51 Cela servira comme toujours à séparer un peu plus le sud musulman des autres provinces du Sud. Pourtant, il existe un fonctionnement régional qui inclut les Chinois, les Thaïs et les minorités (Ivanoff 2008 ; Ferrari 2008) et même les travailleurs illégaux. Ce fonctionnement à portée séparatiste ne fera que renforcer une identité infrarégionale qui ne sera qu'une tentative de balkanisation de la région musulmane pour mieux la contrôler. Car, si l'État commence à rationaliser le passage 
transfrontalier, il risque ne de pas avoir les moyens de le contrôler : border-pass, visa, passeport, double nationalité, illégalité acceptée sur les lieux de travail..., les moyens pour faciliter le passage sont infinis.

\section{Conclusion} développement voulant les utiliser pour polycentrer la région (IMG Growth Triangle, Greater Mekhong Subregion...) qui ne font que renforcer les réseaux maffieux et les trafics locaux d'êtres humains. Cette demande de développement autour de noyaux identifiés par des organismes de développement provoque des réactions et nécessite des redistributions des migrations. Mais, qui peut acheminer les millions de personnes nécessaires à la construction des rêveries idéologiques de ces penseurs du marché ? On retrouve alors un discours sur un monde en mouvement libre de ces entraves (c'est-àdire constitué d'hommes prisonniers du marché) mais autorisant toutes les dérives esclavagistes qui en découlent concrètement. Le système idéologique mis en place provoque une réaction en chaîne des réseaux de trafics humains (Birmans en Thaïlande) et de redéfinitions des politiques économiques de pays qui y voient l'occasion de régler quelques déséquilibres culturels et démographiques internes (Javanais versus Dayak ou Kinh versus Montagnards par exemple). On crée ensuite des commissions des droits de l'homme, des projets de lutte contre le trafic, on exige des pratiques de bonne gouvernance, bref on implémente une idéologie occidentale en laissant aller les migrations spontanées ou trafiquées et manipulées par les élites.

Mentionnons une migration internationalisée qui est à l'origine de la diaspora des Hmong, des réseaux de prostitution sud-est asiatique au Japon, des travailleurs musulmans dans les Émirats... Nous souhaitons également souligner une autre 
migration sur laquelle nous ne pouvons nous étendre, liée au tourisme permmettant de faire " passer » de nombreux travailleurs et prostituées derrière son flux incessant et aux technologies nouvelles de communication favorisant un indigénisme qui espère retrouver une mobilité et une identité transfrontalières à travers les outils modernes.

Les résiliences des mobilités traditionnelles des populations transfrontalières et nomades sont importantes. Nous sommes encore loin des traversées de l'Amazonie par des populations entières aux visions millénaristes se déplaçant sur des milliers de kilomètres. Cependant, on note qu'une partie des échanges traditionnels se fait maintenant avec une vision plus "internationaliste» de la part de ces populations nomades. Mais, on tente de les sédentariser (même si nous ne parlons que de quelques centaines d'individus, Mlabri et Moken en Thaïlande par exemple). La mobilité idéologique et le choix des nomades sont considérés comme un danger en soi par les ethnies dominantes. Le nomadisme offre une vision d'un homme libre que ne peut tolérer une idéologie libérale et centrale voulant inféoder les mouvement de populations à des critères socioéconomiques quels que soient les dommages humains qu'elle provoque. Pourquoi donc quelques milliers de Moken qui traversent la frontière birmano-thaïe inquiètent-ils tant les autorités ? Le problème de la double nationalité, que l'on affirmait ne pas en être un, commence à se poser (voir le Premier ministre malaysien) car la peur de la "marée brune » est réelle. Ainsi, ces nomades sont les avant-postes d'une possible "invasion» des Birmans venus chercher une part du bonheur libéral en Thaïlande. Il faut contenir ce mouvement. Or, la liberté de mouvement aux frontières et celle des nomades sont de très mauvais exemples. C'est pourquoi le nombre de projets visant à la sédentarisation de ces populations est incroyablement supérieur à leur réel enjeu économique et politique. Ils posent une question identitaire vitale. La Thaillande ne peut s'ouvrir à ses voisins d'une manière trop visible. Donner des papiers et reconnaître les migrants ouvrirait la porte à une « invasion » politiquement fantasmée.

Dans le même ordre d'idées, on constate que les Birmans sont cantonnés dans des ghettos où l'on autorise certaines ONG à leur donner des cours et à les soigner dans une certaine limite. Les enfants ont le droit à l'éducation selon la constitution thaïlandaise, alors on les éduque. On les soigne mais on ne veut pas construire d'hôpitaux de peur que les Birmans, qui arrivent en grand nombre, apportent des maladies. On a ainsi mis en place un système de limitation et de contrôle de la migration birmane, illégale tout en étant nécessaire. Cela a un double avantage : une main-d'œuvre servile que l'on peut utiliser et jeter comme on veut mais, aussi, un rendement maximum de la filière.

Il faut enfin insister sur les résiliences culturelles qui, loin d'être des résidus ethniques folklorisés, sont bien plus la marque d'archaïsmes toujours moteurs des déplacements de populations. Ces accords locaux et régionaux permettent la mobilité contrairement aux grandes politiques, aux grands projets et aux soi-disant volontés de mettre un frein aux migrations et aux trafics humains. Les frontières sont incontrôlables et les nations n'ont eu de cesse que de les coloniser, mais en y générant des réseaux souterrains et maffieux, et des résistances culturelles. 


\section{BIBLIOGRAPHIE}

AMARA, P. 1995. «International migrant workers in Asia : The case of Thailand », in Proceedings of the International Conference on Transnational Migration in the Asia-Pacific Region: Problems and Prospects, 1-2 December 1994. Institute of Asian Studies, Chulalongkorn University.

ANDERSON, B. 1991 [1986]. Imagined Communities : Reflections on the Origin and Spread of Nationalism. Londres, Verso Books, 244 p.

APMRN, Migration Issues in the Asia Pacific Introduction Welcome Speech - First Annual Meeting of the APMrn Aotearoa/New Zealand Australia Fiji Hong Kong Indonesia Malaysia People's Republic of China Philippines Republic of South Korea Singapore.

APPADURAI, A. 1996. Modernity at Large : Cultural Dimensions of Globalization. Minneapolis, University of Minnesota Press.

ARCHAVANITKUL, K. 1995. An Overview of Transnational Migration in Thailand: Policy and Areas of Research, Institute of Population and Social Research, Mahidol University (in Thai), Asian Research Center for Migration.

ARCHAVANITKUL, K. 1995. Proceedings of Conference on Transnational Migration in the Asia-Pacific Region : Problems and Prospects, December 1-2, 1994. Institute of Asian Studies, Chulalongkorn University.

ASIA WATCH. 1993. A Modern Form of Slavery: Trafficking of Burmese Women and Girls into Brothel in Thailand. Asia Watch, Women's Rights Project, Division of Human Rights Watch, 1993, 160 p.

Asian Research Center for Migration (АRCM) in collaboration with the World Vision of Thailand. 2004. « Cross Border Labor Migration and Human Trafficking in Ranong ", in Research project Migration and Deception of Migrant Workers in Thailand.

ASIAN DEVELOPMENT BANK. 1999. Policy on Indigenous People, ADB, Manille.

AUGÉ, M. 2009. Pour une anthropologie de la mobilité. Paris, Payot \& Rivages (Manuels Payot), 96 p.

BARTH, F. 1969. Ethnic groups and Boundaries. The Social organization of Culture Difference, Oslo, Scandinavian University et Londres, Allen and Unwin (trad. française in Ph. POUTIGNAT et J. STREIFFFENARD [dir.], Théories de l'ethnicité, Paris, Presses universitaires de France, 1995 : 203-249).

BENJAMIN, G. 1988. «In the long term : Three themes in Malayan cultural ecology », in K. L. HUTTERER, A. T. RAMBo et G. LOVELACE (eds.), Cultural Values and Tropical Ecology in Southeast Asia. Ann Arbor, University of Michigan, Center for South and Southeast Asian Studies (Michigan Papers on South and Southeast Asia ; 27) : 219-278.

BOUTRY, M. 2004. « Un système symbolique en construction : l'exemple du bateau birman », Techniques et Culture, $\mathrm{n}^{\circ}$ 43-44, Mythes. L'origine des manières de faire : 261-276.

BOUTRY, M. 2007. L'archipel Mergui, Croisée des Mondes. Dynamiques d'appropriation du territoire et expression identitaire des pêcheurs birmans. Thèse de doctorat non publiée, Paris, École des hautes études en sciences sociales, $453 \mathrm{p}$.

BOUTRY, M. et O. FERRARI. 2009. Des catastrophes naturelles au désastre humain. Conséquences et enjeux de l'aide humanitaire après le tsunami et le cyclone Nargis en Thailande et Birmanie. Bangkok, IRASEC (Carnets de l'IRASEC $\left.\mathrm{n}^{\circ} 10\right), 120 \mathrm{p}$. 
BOUTRY, M. et J. IVANOFF. 2008. « De la segmentation sociale à l'ethnicité dans les suds péninsulaires? Réflexions sur les constructions identitaires et les jalons ethniques à partir de l'exemple des pêcheurs birmans du Tenasserim ", Aséanie, Sciences humaines en Asie du Sud-Est, décembre : 11-46.

BOUTRY, M. et J. IVANOFF. 2009. La monnaie des frontières. Migrations birmanes dans le sud de la Thailande, structure des réseaux et internationalisation des frontières. Bangkok, IRASEC (Carnet de L'IRASEC, Série observatoire $\mathrm{n}^{\circ}$ 2, Irasec/Observatoire des trafics illicites), $161 \mathrm{p}$.

BRAIDWOOD, R. et C. A. REED. 1957. « The achievement and early consequences of food-production : A consideration of the archeological and natural-historical evidence ", in Cold Spring Harbor Symposia on Quantitative Biology, XXII. New York, The Biological Laboratory Cold Spring Harbor : 19-31.

BRAUDEL, F. [1949] 1966. La Méditerranée et le monde méditerranéen à l'époque de Philippe II. Paris, Armand Colin, deuxième édition révisée.

BRAUDEL, F. 1977. Écrits sur l'histoire. Paris, Flammarion.

BROMBERGER, Ch. et A. MOREL (éd.). 2000. «Trouver la bonne distance », in Limites floues, frontières vives. Des variations culturelles en France et en Europe. Paris, Éditions de la Maison des sciences de l'homme, Mission du Patrimoine ethnologique (Ethnologie de la France, Cahier $n^{\circ} 17$ ) : 27-40. BROWN, D. 1994. The State and Ethnic Politics in South-East Asia. Londres, Routledge, 354 p. BRUN, P. et P. de MIROSCHEDJI. 1998-1999. Cahiers des thèmes transversaux ArScAn, Cahier I Avantpropos au thème 2 et Thème 2 - Évolution des structures et dynamiques sociales : 49-52.

CONDOMINAS, G. 1978. « A few remarks about Thai political systems, » in G. B. MILNER (ed.), Natural Symbols in South East Asia. Londres, University of London, School of Oriental and African Studies : 105-112.

CONDOMINAS, G. 1980. L'espace social. À propos de l'Asie du Sud-Est. Paris, Flammarion (Sciences), $539 \mathrm{p}$.

CONDOMINAS, G. (co-dirigé avec J. IVANOFF et M.-A. MARTIN). 1998. Formes extrêmes de dépendance en Asie du Sud-Est. Contributions à l'étude de l'esclavage. Paris, Éditions de l'EHEss (Civilisations et Sociétés ; 96), $582 \mathrm{p}$.

CORBIN, A. 1988. Le territoire du vide. L'occident et le désir du rivage (1750-1840), Paris, Aubier. DUNCAN, Ch. R. (ed.). 2008. Civilizing the Margins. Southeast Asian Government Policies for the Development of Minorities. Singapour, NUS Press, $284 \mathrm{p}$.

FERGUSON, J. 1994. The Anti-politics Machine. "Development", Depoliticization, and Bureaucratic Power in Lesotho. Mineapolis, University of Minesota Press, $336 \mathrm{p}$.

FERRARI, O. 2008. « Ritual structure and coordinates of the Moklen nomadism : Towards an understanding of the social integration of maritime societies in Southern Thailand ", in Southern Ethnic Dynamism : the Andaman littoral and marine populations. CUSRI (Chulalongkorn University Social Research Institute)/IRASEC (Institut de recherche sur l'Asie du Sud-Est contemporaine) : 75-94.

FERRARI, O. 2009. « How to be Thai and Sea Gypsies? Technical, cultural and ritual syncretism », Actes du colloque Chao Lay : Ethnic Dynamism and Cultural Rivitalization, Siridhorn Anthropology Center/Cusri/Irsec, Ambassade de France), 2009, p. 30-42. 
FERRARI, O., A. LEVEAU, N. HINSHIRANAN et J. IVANOF. 2010. Thailande. Aux origines d'une crise. Bangkok, IRASEC (Carnets de L'IRASEC $\left.\mathrm{n}^{\circ} 13\right)$.

GODELIER, M. 2007. Au fondement des sociétés humaines. Ce que nous apprend l'anthropologie. Paris, Albin Michel (Bibliothèque Idées), 293 p.

GODELIER, M. 2009. Communauté, société, culture. Trois clefs pour comprendre les identités en conflits. Paris, CNRS Éditions, 60 p.

GUÉRIN, M., A. HARDY, N. VAN CHINH, S. T. B. HWEE. 2003. Des montagnards aux minorités ethniques. Quelle intégration nationale pour les habitants des hautes terres du Viêt Nam et du Cambodge? Paris, Bangkok, L'Harmattan/IRASEC (Analyses en regard), 354 p.

HNIN HNIN, P.1988. Aids and Prostitution in Thailand : Case Study of Burmese Prostitutes in Ranong. Master of City Planning, Massachussets Institute of Technology, $64 \mathrm{p}$.

HORSTMANN, A. 2002a. «Incorporation and resistance : borderlands, transnational communities and social changing Southeast Asia ", Working Paper WPTC-02-04, Oxford, ESRC Transnational Communities Program, $27 \mathrm{p}$.

HORSTMANN, A. 2002b. « Trapped Ethnic Minorities and Local Reworking of Citizenship at the Thailand-Malaysian Border », Working Paper, Center for Border Studies, Queens University of Belfast.

HORSTMANN, A. 2002c. Class, Culture and Space. The Construction and Shaping of Communal Space in Southern Thailand. Bielefeld, Transcript Verlag (Culture and Social Practice), 175 p.

horstmann, A. 2006. «States, Peoples, and Borders in Southeast Asia », in Articles. Kyoto Review of Southeast Asia, $\mathrm{n}^{\circ} 7: 1-9$.

HORSTMANN, A. et L. W. REED (eds.). 2005. Centering the Margin Agency and Narratives in Southeast Asian Borderlands (Anthropology of Asia Series). Oxford Berghahn Books, $248 \mathrm{p}$.

HUTTERER, K. L., A. T. RAMBO, G. LOVELACE (eds.). 1988. Cultural Values and Tropical Ecology in Southeast Asia. Ann Arbor, University of Michigan, Center for South and Southeast Asian Studies (Michigan Papers on South and Southeast Asia ; 27), 417 p.

IVANOFF, J. 2003. « La mort de l'ethnologie ? Réflexions à partir de l'ouvrage Ethnologie. Concepts et aires culturelles (Paris, Armand Colin, 2001, 320 p.) », Techniques et Culture, 41 : 169-190.

IVANOFF, J. 2004. Les naufragés de l'histoire. Les jalons épiques de l'identité moken. Paris, Les Indes Savantes, $593 \mathrm{p}$.

IVANOFF, J. 2008a. « Ethnoregionalism and ethnic boundaries in Southern Thailand », in Southern Ethnic Dynamism : the Andaman littoral and marine populations, Cusri (Chulalongkorn University Social Research Institute)/Institut de recherche sur l'Asie du Sud-Est contemporaine (Irasec) : 2-19.

IVANOFF, J. 2008b. « Change, resistance or cultural permanence among the Sea Faring Populations ", in Southern Ethnic Dynamism : the Andaman littoral and marine populations, Cusri (Chulalongkorn University Social Research Institute)/Institut de recherche sur l'Asie du Sud-Est contemporaine (Irasec) : 95-112.

IVANOFF, J. 2009. «Resilience of Sea Gypsies culture and new indigenous future », Actes du colloque Chao Lay : Ethnic Dynamism and Cultural Rivitalization, Siridhorn Anthropology Center/ Cusri/Irasec, Ambassade de France : 43-67. 
IVANOFF, J. 2010.The Cultural Roots of Southern Violence in Malay Southern Thailand. Comparative Mythology. 1. The Tutelary Figure of Malay Political Heroism. Bangkok, White Lotus, 333 p. (le volume 2, The Sociopolitical and Imaginary Structures of the Southern Rebellion est à paraitre en 2011, Irasec/White Lotus).

JAGGAN, L. 2009. Spectrum, vol. 2, n 7, Bangkok Post : 3-5.

KEYES, Ch. 2008. « Ethnicity and the Nation-States of Thailand and Vietnam », in P. LEEPREECHA, D. MCCASKILL et KWANCHEWAN BUADAENG (eds.), Challenging the limits. Indigenous Peoples of the Mekong Region. Chiang Mai, Mekong Press Foundation : 13-53.

KARDINER, A. [1939] 1969. L'individu dans la société. Paris, Gallimard.

LeEPREECHA, P., D. MCCASKILl et KWANCHEWAN BUADAENG (eds.). 2008. Challenging the Limits. Indigenous Peoples of the Mekong Region. Chiang Mai, Mekong Press, 379 p.

LEVEAU, A. (dir.). 2009. L'Asie du Sud-Est 2009. Les événements majeurs de l'année. Bangkok, IRASECLignes de repères (Documents), $310 \mathrm{p}$.

Loos, T. 2006 [2002]. Subject Siam. Family, Law, and Colonial Modernity in Thailand. Bangkok, Silkworms Books, $212 \mathrm{p}$.

MURSHID, K. A. S., T. SOKPHALly et al. 2005. The Cross Border Economies of Cambodia, Laos, Thailand and Vietnam. Development Analysis Network.

PAVIN, C. 2005. A Plastic Nation. The Curse of Thainess in Thai-Burmese Relations. Lanham, Univeristy Press of America, $188 \mathrm{p}$.

PAUL, S. R. et al. 1997. Reproductive Health Survey of Migrant Burmese Women in Ranong Fishing Community, Thailand, in $12^{\text {th }}$ World Conference of the Society for International Development in Santiago, Spain, 1997.

PRESCOTT, J. R. 1987. Political Frontiers and Boundaries. Londres, Unwin Hyman, 210 p.

PRESS, B. 2004. Untangling vulnerability. A Study on HIV/AIDS Prevention Programming for Migrant Fishermen and Related Populations in Thailand. Bangkok, Raks Thai Foundation, 120 p.

PRIES, L. 2000. « Transnational social space : Do we need a new approach in response to new phenomena? », in P. LUDGER (ed.), New Transnational Social Spaces. International Migration and Transnational Companies. Londres, Routledge.

PREMJAI, V., S. AUASALUnG et S. CHANTAVANICH. 1999. Migrant Children in Difficult Circumstances in Thailand. Bangkok, Asian Research Center for Migration, Institute of Asian Studies, Chulalongkorn University.

RAJAH, A. 1990. «Ethnicity, nationalism and the nation-state : the Karen in Burma and Thailand », in G. WIJEYEWARDENE (ed.), Ethnic Groups Across National Boundaries in Mainland Southeast Asia. Singapour, Institute of Southeast Asian Studies : 102-133.

REYNOLDS, G. J. 2002. «Introduction : national identity and its defenders : Thailand Today », in G. J. REYNOLDS (ed.), National Identity and its Defenders : Thailand Today. Chiang Mai, Silkworms Booksm Thailand, 2002, p. 1-32.

scotT, J. 2009. The Art of Not Being Governed : An Anarchist History of Upland Southeast Asia. New Haven, Yale University Press.

ScoTT, J. 1998. Seeing Like a State: How certain schemes to improve the human condition have failed. New Haven, Yale University Press. 
scoTt, J. 1990. Domination and the Arts of Resistance : Hidden Transcripts. New Haven, Yale University Press.

scotT, J. 1985. Weapons of the Weak : Everyday Forms of Peasant Resistance. New Haven, Yale University Press.

ScoTT, J. 1979. The Moral Economy of the Peasant : Rebellion and Subsistence in Southeast Asia. New Haven, Yale University Press.

SUDTHICHIT, C., G. RISSER et S. CHANTAVANICH. 1997. The Monitoring of the Registration of Immigrant workers from Myanmar, Cambodia and Laos in Thailand. Bangkok, Asian Research Center for Migration, Institute of Asian Studies, Chulalongkorn University.

SUPANG, C. et M. BUNNA, «Thai national approaches to the legal protection of women migrant workers », Asian Migrant, vol. 10 (4) : 110-113.

SUPANG, C., S. PAUL et al. 2000. Cross-border Migration and HIV Vulnerability in the Thai-Myanmar Border. ARCM, Chulalongkorn University, mars.

SUPANG, C. et al. 2000. Cross-border Migration and HIV/AIDS Vulnerability at the Thai-Cambodia Border : Sangkhlaburi and Ranong. Bangkok, Asian Research Center for Migration, Institute of Asian Studies, Chulalongkorn University.

SUPANG, C., P. VUNGSIRIPHISAI et S. LAODUMRONGCHAI. 2003.Research Report on Migration and Deception of Migrant Workers in Thailand. Bangkok, World Vision Foundation of Thailand (WVFT) in collaboration with Asian Research Center for Migration (ARCM).

SUPANG, C., P. VUNGSIRIPHISAI et S. LAODUMRONGCHAI. 2007.Thailand Policies towards Migrant Workers from Myanmar. Bangkok, Asian Research Centre for Migration, Institute of Asian Studies, Chulalongkorn University, $82 \mathrm{p}$.

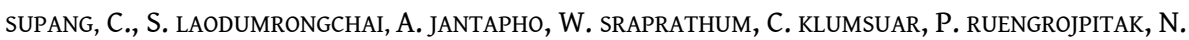
EKSAENGSRI, T. WONGBOOMCHAINAN, W. JITPONG. 2007.Mitiging Exploitative Situations of Migrant Workers in Thailand. Bangkok, The Asian Research Center for Migration, Institute of Asian Studies, Chulalongkorn, $200 \mathrm{p}$.

SUPANG, C. et al. 2000. Étude de la pénurie de main-d'œuvre en Thailande en 1999 (en thaï). Bangkok, Asian Research Center for Migration, Institute of Asian Studies, université Chulalongkorn.

SUPANG, C. et al. 2000. Cross-border Migration and HIV/AIDS Vulnerability at the Thai-Cambodia Border : Aranyaprathet and Khlong Yai. Bangkok, Asian Research Center for Migration, Institute of Asian Studies, Chulalongkorn University.

SUPANG, C. et G. RISSER. 1995. National Policy and Crossborder Migration in the Asia-Pacific : Security and Social Implications, Paper presented at the Ninth Asia Pacific Roundtable, Kuala Lumpur, 5-9 June. SUPANG, C. et G. RISSER. 1995. Thai Migrant Worker in Southeast and East Asia: The Prospects of Thailand's Migration Policy in the Light of the Regional Economic Recession. Bangkok, Asian Research Center for Migration, Institute of Asian Studies, Chulalongkorn University.

SUPANG, C. et G. RISSER. 1996. « People on the move : Issues and areas of potential research for transnational migration in Thailand and Asia », Journal of Social Research, vol. 19 (1) : 114-129. SUPANG, C. 1994. « Refugees from Myanmar and Indochina », in Asia Yearbook 1994, Institute of Asian Studies, Chulalongkorn University.

SUPANG, C. 1995. Crossborder Migration in Asia-Pacific and Labour Linkages, Working paper, Institute of Asian Studies, Chulalongkorn University. 
SUPANG, C. 2001. « Mouvements de population en Thaïlande, deux décennies d'incertitudes et de problème non résolus ", in S. DOVERT (dir.), Thailande contemporaine. Bangkok, IRASEC

(Monographies nationales) et Paris, L'Harmattan : 248-273.

SUTHEP, C., Spectrum, vol. 2, n 7, Bangkok Post, 2009 : 8-9.

TAYLOR, R. 1987. The State of Burma, London, C. Hurst \& Company, 1987, 395 pp.

THONGCHAI WINICHAKUL. [1994] 2005. Siam Mapped. A history of the Geo-Body of a Nation. Chiang Mai, Silkworm books, 228 p. (1 ${ }^{\text {re }}$ édition University of Hawaii Press).

TO XUAN, P. 2008. « Whose land, whose forest ? Contesting Highland forest resources in Vietnam », in P. LEEPREECHA, D. MCCASKILl et KWANCHEWAN BUADAENG (eds.), Challenging the Limits. Indigenous

Peoples of the Mekong Region. Chiang Mai, Mekong Press : 77-115.

TRÉGLODÉ, B et A. LEVEAU (dir.). 2010. L'Asie du Sud-Est 2010. Les événements majeurs de l'année. Bangkok, IRASEC-Lignes de repères (Documents), $379 \mathrm{p}$.

WICKRAMASEKARA, P. 1995. Recent Trends in Temporary Labour Migration in Asia, Paper presented at the International Seminar on Migration and the Labour Market in Asia in the Year 2000, Tokyo, 19-20 January 1995.

WIJEYEWARDENE, G. (ed.). 1990. Ethnic Groups Across National Boundaries, in Mainland Southeast Asia. Singapour, Institute of Southeast Asian Studies (Social issues in Southeast Asia).

WIJEYEWARDENE, G. 2002. « The frontiers of Thailand », in G. J. REYNOLDS (ed.), National Identity and its Defenders : Thailand Today. Chiang Mai, Silkworm Books : 126-151.

WOLTERS, O. W. 1999. History, Culture, and Religion, in Southeast Asian Perspectives. Édition révisée, Ithaca, New York, Cornell University (Southeast Asian Program Publications n²6), 275 p.

WONGBOONSIN, P., T. SUPAPHAN, T. SAIKAEW, S. LAODUMRONGCHAI, Y. NUTTAPOJ. 2008. Trafficking for sexual exploitation into southern Thailand. Bangkok, IRASEC/IAS (Occasional Paper $\left.n^{\circ} 4\right), 82 \mathrm{p}$.

\section{NOTES}

i. Thierry Lejard, dans sa présentation à la conférence Asian Borderlands : Enclosure, Interaction and Transformation (5 novembre 2010, Chiang Mai), remarque que cette ligne de camps arquait la frontière et donc l'échange entre les Thaïlandais, les organismes internationaux et les combattants Khmers rouges puis les Khmers tout court.

ii. Une équipe s'est formée sur la thématique de la dynamiques ethnique et sociale des frontières avec Olivier Ferrari, Thierry Lejard, Narumon Hinshirnan, Jacques Ivanoff, Maxime Boutry en collbration avec L'IRASEC, les universités thaïlandaises de Chulalongkorn et Chiang Mai. Nous avons participé sur ce sujet au $3^{e}$ congrès du Réseau Asie, en 2007, et à sa publication, en 2010, à la la conférence Asian Borderlands: Enclosure, Interaction and Transformation, le 5 novembre 2010 à Chiang Mai (panel Identity Construction in the Borderlands), aux Burma Studies en 2010 (panel sur les Moving Borders of Burma), nous collaborons à un ouvrage sur les Peuples de frontières à paraître en 2011 (avec Frédéric Bourdier et Christian Culas), à un projet de recherche collectif et comparatif Settling Process and Transition among three Nomadic Populations of Thailand(un projet de recherché mis en œuvre par CESD et IRD avec la participation de l'IRASEC) pour 2010-2011, nous travaillons également sur les migrations illégales dans le cadre d'un projet développé avec l'Observatoire des trafics illicites Les portes de l'illégalité en 2011, enfin, nous prévoyons un panel pour le $4^{\mathrm{e}}$ congrès du réseau Asia avec Nathalie Fau sur les Villes-doublons prévu en 2011.

iii. Par Malaisie nous entendons la Malaysia actuelle et Singapour. 
iv. Indispensables au développement du sud, les "courtiers", pueza, sont indissociables du marché économique que représente l'immigration illégale. Le terme de courtier implique la notion de vente et suggère d'emblée le trafic d'êtres humains, difficile à définir avec précision dans le flot de l'immigration illégale consentante ou spontanée tout autant que de l'en différencier. Notons tout d'abord que le terme même de pueza ne se limite pas au champ de l'immigration, mais est utilisé pour toute tractation commerciale faisant appel à un courtier ou un intermédiaire. Par ailleurs, il est utilisé dans l'ensemble de la Birmanie et également dans le cadre d'échanges interethniques

v. Patron et entrepreneur, un mot d'origine chinoise. C'est un personnage dominant et très fortement présent en Thaillande du sud. Il est l'homme à qui l'on emprunte, auprès de qui l'on s'endette mais, aussi, auprès de qui on cherche protection et qui se charge des relations commerciales entre nomades et sédentaires..

vi. En 2009 des Rohingyas ont été rejetés à la mer par la marine thaïlandaise. En dépit des démentis, des commissions d'enquêtes ont été créées et ont révélé la véracité du problème que nous avons nous-mêmes observés (Boutry et Ivanoff 2009). Mais, ces centaines de Rohingyas, venus de Birmanie et parfois du Bangladesh où ils ont cherché refuge car ils ne sont pas reconnus en Birmanie, étaient beaucoup plus que cela. Il s'agit de milliers de personnes qui cachaient un phénomène beaucoup plus préoccupant, celui des millions de Birmans qui colonisaient le sud de la Thaillande. Pour finir des commissions ont été créées, les Birmans ont accepté de reprendre les Rohingyas qui pouvaient justifier de leur nationalité et les quelques centaines de cas répertoriés ont permis de cacher la réalité de milliers d'autres Rohingyas mais surtout des millions de Birmans (Boutry et Ivanoff 2009).

vii. Il serait trop long d'entrer dans les détails, mais citons par exemple les volontés d'intégration de populations du nord de la Thaïlande, l'ethnicité fidèle et vacillante des Karen, l'actuelle action du gouvernement thaïlandais qui veut regrouper les Chaolay, les nomades marins, c'est-à-dire influencer sur leur identité. Les autorités ne font que permettre aux nomades de renouer des contacts avec des cousins oubliés (voir Southern Ethnic Boundaries in Southern Thailand,actes des colloques de Chulalongkorn 2008 et Chao Lay : Ethnic Dynamism and Cultural Rivitalization, Sirindhorn Anthropology Center, 2009).

viii. Les Moken de Birmanie devant la difficulté d'accomplir leurs rituels communautaires, leur exogamie de groupes et leurs constructions navales sont dans une position d'attentisme. La société réfléchit et attend le grand chamane qui leur montrera la voie à suivre pour l'adaptation. Si de 150 bateaux en 2003, on passe de 20 bateaux véritablement nomades en 2007, il ne s'agit pas d'un signe de la disparition de l'ethnie mais de sa transformation, de son adaptation, même si celle-ci passe par la mixité avec les Birmans ou la création de nouveaux rituels. Il s'agit bien d'un mouvement millénariste, celui d'une population qui demande une nouvelle règle pour s'adapter, et déjà des pionniers sont à l'œuvre pour tenter de répondre aux interrogations.

ix. En ce qui concerne le ratio autorisé officiel de $1 / 9$, notons simplement que, sur les bateaux enregistrés à Ranong, il est en réalité de 1/12.

$\mathbf{x}$. Le terme Isan, d'origine Pali-Sanskrit, veut dire Nord-Est et ne contient aucune signification impliquant une segmentation ou voie vers l'autonomisation. Le Nord-Est n'existe que parce qu'il a été créé par les colonisateurs occidentaux.

xi. «Invité » et par extension les Indiens et musulmans considérés comme populations allogènes à la Thaillande.

xii. « Homme Malais » en langage de Patani (un dialecte du Malais proche de celui du Kelantan).

xiii. UMIFRE 22, projet Borders and Mobility.

xiv. Peuples de frontières (F. Bourdier, C. Culas, J. Ivanoff), IRASEC (à paraître). 


\section{RÉSUMÉS}

Les mouvements multiformes de populations en Asie du Sud-Est, sont le résultat de guerres, de tracés de frontières, de conflits ethniques... Réfugiés, immigrés (légaux et illégaux) ou déplacés, on ne compte plus les problèmes des États de la région pour gérer les flux de populations, notamment transfrontaliers. Ces derniers, de plus en plus importants, résultent de la conjonction de plusieurs facteurs, notamment l'ouverture des pays au commerce, la volonté de « régionaliser » l'Asie du Sud-Est et les déséquilibres économiques entre les pays. Pourquoi et comment les frontières modernes facilitent ces mouvements de populations, de quelques milliers de nomades vivant sur les frontières de plusieurs pays à des millions de travailleurs illégaux venus chercher un avenir dans un pays d'accueil. Par exemple, en dépit de l'imposition de règles administratives de plus en plus contraignantes, la porosité des frontières et les réseaux multiséculaires de certaines régions permettent une "inflation» des mouvements de populations. En trente ans, le nombre de réfugiés et de travailleurs immigrés dans le sud de la Thaillande est passé de quelques centaines de milliers à plusieurs millions. La question des minorités se pose moins en termes de chiffres que d'identité. Les États-nations de la région n'arrivent pas à intégrer leurs populations frontalières. Les questions essentielles - qui est citoyen de tel ou tel pays et selon quels critères - sont loin d'être résolues. Les agences nationales et les Ong ont mis en œuvre des moyens pour gérer le flux et l'ambiguïté délibérément maintenue par les gouvernements sur des statuts des réfugiés et des travailleurs illégaux pour contrôler, selon leurs besoins, ces millions de personnes. Quels sont les facteurs déterminants qui entraînent ces déplacés vers des rêves souvent illusoires et dans des filières de plus en plus internationales? Comment ces populations des frontières se développent-elles, construisentelles de nouveaux espaces " pionniers", de nouvelles identités ou de nouvelles pratiques sociales à travers toute l'Asie du Sud-Est? Car, le phénomène de la «birmanisation » du sud de la Thaïlande et l'extension des réseaux des trafics ne sont pas uniques à cette région et peuvent être comparés à d'autres situations similaires observées ailleurs. Existe-t-il une structure commune contemporaine à ces déplacements?

Wars, shifting borders, ethnic conflicts and other factors all cause multiform population movements in South-East Asia. Refugees, immigrants (legal and illegal) and displaced persons are only a few of the problems this region faces when managing population flows, especially across borders. These growing cross-border movements are linked to several factors, including the opening up to trade, the desire to "regionalise" South-East Asia and economic differences between countries. How and why do modern borders encourage population movements - from the thousands of nomads living in border areas to the millions of illegal workers seeking new futures in host countries? For example, despite increasingly strict administrative rules, some regions' porous borders and ancient networks have led to "inflationary" population movements. Thus, the number of refugees and immigrant workers in southern Thailand has jumped from several hundred thousand to several million in 30 years. Minority groups are no longer merely an issue because of their number, but also because of their identity. Nation states in the region are unable to integrate border populations. Fundamental questions - who is a citizen of what country and based on which criteria - remain unanswered. National agencies and NGOs have implemented measures to manage both these flows and governments' ambiguous approaches towards refugees and illegal workers, which allow them to control millions of people to satisfy their own needs. What leads displaced people towards what are often illusory dreams and increasingly international networks? How do border populations develop, build "pioneering" areas, take on new identities and adopt new social practices in South-East Asia? The "Burma- 
isation" of southern Thailand and widening trafficking networks are not unique to this region, and can be compared to similar situations observed elsewhere. Are these movements characterised by a common contemporary structure?

INDEX

Index géographique : Asie du Sud-Est, Thaillande, Birmanie

Mots-clés : flux migratoires, transfrontaliers, identité, statut des réfugiés

Keywords : migration, transborder, identity, status of refugees, mobility, Southeast Asia, Thailand, Burma

Thèmes : migration, mobilité

\section{AUTEUR}

\section{JACQUES IVANOFF}

Ethnologue, membre de l'Usr 3142 de l'Institut de recherche sur l'Asie du Sud-Est contemporaine (Irasec-umifre22,http://www.irasec.com/) depuis 2008.

ivanoffjacques@yahoo.com 